\title{
Assessment of salivary free cortisol levels by liquid chromatography with tandem mass spectrometry (LC-MS/MS) in patients treated with mitotane
}

\author{
Cinzia Carrozza, ${ }^{1}$ Rosa Lapolla, ${ }^{1}$ Jacopo Gervasoni, ${ }^{1}$ Carlo Antonio Rota, ${ }^{2}$ \\ Pietro Locantore, ${ }^{2}$ Alfredo Pontecorvi, ${ }^{2}$ Cecilia Zuppi, ${ }^{1}$ Silvia Persichilli ${ }^{1}$ \\ ${ }^{1}$ Institute of Biochemestry and Clinical Biochemestry, ${ }^{2}$ Institute of Endocrinology, Università Cattolica del Sacro \\ Cuore, Rome, Italy
}

\begin{abstract}
OBJECTIVE: Mitotane is an adrenocytolytic agent used in adrenocortical carcinoma, inducing adrenal insufficiency, requiring replacement treatment. Such therapy is not easy to monitor because of mitotane interference. Salivary cortisol reflects a free fraction of plasma cortisol and may be useful in such patients. DESIGN: The aim of our study was to evaluate salivary cortisol by HPLC coupled to tandem-mass spectrometry (LC-MS/MS) and by an electrochemiluminescence immunoassay (ECLIA) in patients treated with mitotane. We enrolled 6 patients receiving mitotane and 2 Addison disease patients as negative controls and determined salivary cortisol rhythm. We also determined the salivary cortisol rhythm in 8 healthy subjects. Salivary samples $(n=112)$ were assayed by ECLIA, using Roche Modular E170, and by LC-MS/MS. RESULTS: The mean values obtained by ECLIA were significantly higher than those obtained by LC-MS/MS in the mitotane group (p<0.001). In fact, in the group measured by LC-MS/ MS, we observed several peaks eluting at a retention time different from the cortisol group, presumably due to cortisol-like analogues. In Addison disease, since steroidogenesis is absent, salivary cortisol values measured by the two methods did not show any significant difference $(p=0.61)$. CONCLUSIONS: Salivary cortisol measured by LC-MS/MS is a selective method, excluding cortisol analogues accumulating in treated patients. Therefore, LC-MS/MS offers an effective system to monitor replacement therapy in mitotane treated patients.
\end{abstract}

Key words: Adrenocortical carcinoma, LC-MS/MS, Mitotane, Salivary cortisol

Address for correspondence:

Cinzia Carrozza, Catholic University School of Medicine,

Largo A Gemelli 8, Rome 00168, Italy, Tel.: +39-0630154222,

Fax: +39-0630156783, Email: ccarrozza@rm.unicatt.it

Received 12-03-12, Revised 03-05-12, Accepted 15-06-12

\section{INTRODUCTION}

Adrenocortical carcinoma (ACC) is a rare malignancy (incidence 1-2 per million population per year) with a heterogeneous presentation and a variable but generally poor prognosis. ${ }^{1}$ Mitotane is the main drug for ACC treatment and it plays a role both in adjuvant 
treatment after complete resection and in recurrent, inoperable and/or metastatic ACC., ${ }^{2,3}$ Mitotane blocks adrenocortical steroid synthesis by inhibiting cholesterol side-chain cleavage (20, 22-desmolase) and $11 \beta$-hydroxylase. ${ }^{4}$ It also exerts a specific cytotoxic effect on adrenocortical cells and it is the only adrenal-specific agent used for the treatment of ACC as well as for other causes of Cushing's syndrome. ${ }^{5}$ Mitotane induces adrenal insufficiency, requiring hydrocortisone replacement therapy. For this reason, cortisol levels must be frequently monitored to assess therapy. One way to assess replacement therapy is to evaluate the patient's quality of life (QoL), using a specific questionnaire, although QoL can be affected both by the oncological status and by concomitant chemotherapy. Moreover, sodium, potassium, ACTH, serum cortisol as well as urinary free cortisol (UFC) ${ }^{7}$ can be measured. However, serum cortisol cannot be used to monitor treatment efficacy, since mitotane increases cortisol binding globulin (CBG), artificially raising total cortisol; $;, 9$ plasma $\mathrm{ACTH}$ is a pulsatile indirect measure of circulating corticosteroids levels and may remain persistently suppressed in patients with ACC long-standing hypercortisolism. Moreover, mitotane leads to falsely elevated UFC values as it alters steroid clearance (increased catabolism). ${ }^{8}$ Salivary cortisol (SC) is not affected by CBG alterations, and reflects the free serum cortisol and may enable a more accurate evaluation in monitoring steroid replacement therapy in patients on mitotane and, reflecting the free serum cortisol, may allow a more accurate evaluation in monitoring steroid replacement therapy in patients on mitotane. ${ }^{10}$ A comparison between salivary and plasma cortisol in patients on mitotane was first performed by Daffora et al. who demonstrated a discrepancy between saliva and plasma cortisol using a RIA method (Radim, Rome, Italy), such a difference being considered to be due to the
CBG elevation induced by mitotane. ${ }^{11}$

Several immunoassay methods have been proposed to determine SC and various studies show a general overestimation of SC values measured by immunoassays compared with those measured by mass spectrometry. ${ }^{12-15}$

The purpose of our study was to assess SC during the follow-up of patients treated with mitotane using two different methods: the electrochemiluminescence immunoassay (ECLIA), a widely employed method in the clinical laboratory, and high-performance liquid chromatography (HPLC) coupled with tandem mass spectrometry (LC-MS/MS), considered as the reference method.

\section{SUBJECTS AND METHODOLOGY}

We retrospectively evaluated 80 salivary samples from 6 ACC patients ( 4 males and 2 females, mean age $48 \pm 12 \mathrm{yr}$ ) on hydrocortisone replacement therapy treated with mitotane for a mean time of $4 \pm 3$ years, as well as from 2 patients affected by Addison disease (AD) on replacement treatment with hydrocortisone $30 \mathrm{mg} /$ day and fludrocortisone $0.1 \mathrm{mg} /$ day, used as negative controls. At the moment of the analysis all patients on mitotane had mitotanemia levels between 14 and $20 \mathrm{mg} / \mathrm{l}$, within the therapeutic range (mean $\pm \mathrm{SD}$ $16.7 \pm 1.7 \mathrm{mg} / \mathrm{l})$. Saliva samples were collected every 2 hours on the same day from 6:00 to 24:00 (Table 1). We also determined the salivary cortisol rhythm in 8 healthy subjects $(\mathrm{n}=32$ samples $)$ at the following times: 8:00-12:00 - 16:00-24:00. Saliva was collected with a commercially available device (Salivette, Sarstedt; cotton swab without preparation no.51.1534). Salivary samples were centrifuged at $4^{\circ} \mathrm{C}$ for $10 \mathrm{~min}$ and stored at $-80^{\circ} \mathrm{C}$ until assayed. Salivary cortisol was determined by LC-MS/MS with a modified version

Table 1. Values of salivary cortisol (nmol/l) evaluated in 6 patients affected by ACC and in 2 patients affected by Addison disease (AD) determined at different times on the same day by LC-MS/MS $v s$ an ECLIA method

\begin{tabular}{|c|c|c|c|c|c|c|c|c|c|c|c|c|c|c|c|c|c|c|c|c|}
\hline & \multicolumn{2}{|c|}{6.00} & \multicolumn{2}{|c|}{8.00} & \multicolumn{2}{|c|}{10.00} & \multicolumn{2}{|c|}{12.00} & \multicolumn{2}{|c|}{14.00} & \multicolumn{2}{|c|}{16.00} & \multicolumn{2}{|c|}{1800} & \multicolumn{2}{|c|}{20.00} & \multicolumn{2}{|c|}{22.00} & \multicolumn{2}{|c|}{0.00} \\
\hline & LC-MSIMS & ECLIA & MSIMS & ECLIA & MSIMS & ECLIA & MSIMS & ECLIA & MSIMS & ECLIA & MSIMS & ECLIA & MSIMS & ECLIA & MSIMS & ECLIA & MSIMS & ECLIA & 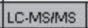 & CLIA \\
\hline atient 1 & 2,207 & 2,588 & 2,207 & 2,000 & 21,517 & 33,655 & 4,414 & 4,993 & 2,759 & 3,669 & 12,138 & 8,634 & 4,965 & 5,407 & 2,207 & 2,298 & 0,552 & 1,539 & 0,828 & 1,688 \\
\hline Satien & 379 & 15,641 & 552 & \begin{tabular}{|c|}
10,096 \\
\end{tabular} & 8,206 & 137,406 & 14,896 & 30,014 & 120,827 & 175,833 & 2,759 & 12,469 & 2,207 & 10,869 & 2,483 & 12,800 & 3,034 & 27,365 & 1,931 & 20,138 \\
\hline Datie & 621 & 6,455 & 2,400 & 5,131 & 0,662 & 73,489 & 3,945 & 11,421 & 10,069 & 18,979 & 7,310 & 14,427 & 421 & 6,648 & 1,986 & 7,007 & 1,324 & 2,869 & 1,407 & 7,007 \\
\hline Patie & 600 & 1,374 & 1,379 & 2,648 & 600 & 31,724 & 2,097 & 10,924 & 9,655 & 23,448 & 5,407 & 6,952 & 3,641 & 5,462 & 4,414 & 6,979 & 1,986 & 4,441 & 1,379 & 1,879 \\
\hline atie & 054 & 1,214 & 3,260 & 17,434 & 8,732 & 35,779 & 4,001 & 11,641 & 58,754 & 57,986 & 4,948 & 9,269 & 3,184 & 7,752 & 4,039 & 3,641 & 0,779 & 2,750 & 0,421 & 6,703 \\
\hline ati & 517 & 15,862 & 379 & 4,414 & 8,413 & 103,172 & 26,344 & 152,551 & 34,758 & 58,234 & 7.724 & 14,538 & 10,759 & 20,276 & 5,069 & 22,676 & 3,862 & 22,731 & 3,862 & 22,069 \\
\hline & 159 & 1,434 & 462 & 1,269 & 10,262 & 16,110 & & 6. & 3,917 & 6,979 & .510 & 3,0 & 1,738 & 1,95 & 3,945 & 5,71 & 1,545 & 1,90 & 1,021 & 1,988 \\
\hline atient 8 & 1,103 & 1,738 & 0,992 & 1,652 & 6,890 & 8,110 & 4,414 & 5,131 & 101,593 & 60,634 & 6,621 & 5,490 & 0,925 & 1,600 & 2,517 & 2,897 & 1,867 & 1,876 & 0,125 & 2,29 \\
\hline
\end{tabular}


of a previously described method ${ }^{16}$ and also assayed using an ECLIA method on Modular Analytics E170 (Roche ${ }^{\circledast}$ Diagnostics GmbH, Mannheim, Germany, www.roche-diagnostics.com), without pretreating the sample, as indicated by the manufacturer. Analytical and functional sensitivities of the method were 0.6 and $1.9 \mathrm{nmol} / \mathrm{L}$, respectively. Intra- and interassay variation coefficient was $<10 \%$. Correlation between variables was evaluated by linear regression analysis and the differences between the two methods were evaluated by Pearson's coefficient; we also performed a paired t-test between ECLIA and LC-MS/MS values. Statistical significance in the results was accepted at a p-value $<0.05$. Statistical analysis was performed using Microsoft Excel 2003 (Microsoft Office 2003). Data are presented as mean \pm standard error (SE).

\section{RESULTS}

The linear regression analysis of the $\mathrm{SC}$ results obtained by LC-MS/MS (x) and ECLIA (y) methods in the $\mathrm{AD}$ group were correlated with a resulting equation $y=1.31 x+0.12(R 2=0.88)$. On the other hand, the mitotane group and healthy subjects were also correlated, even though the resulting equations were $y=1.69 x+3.59(R 2=0.53)$ and $y=1.69 x+5.03$ $(\mathrm{R} 2=0.68)$, respectively. However, the mean cortisol concentration obtained by ECLIA (for the whole set of samples) was significantly higher than the values obtained by LC-MS/MS ( $\mathrm{p}<0.001)$ for patients undergoing mitotane treatment (mean \pm SE $11.84 \pm 1.74$ $\mathrm{nmol} / \mathrm{L}$ vs $4.90 \pm 0.75 \mathrm{nmol} / \mathrm{L}$, respectively) as well as for the healthy subjects group (mean \pm SE $10.88 \pm$ $1.16 \mathrm{nmol} / \mathrm{L}$ vs $3.47 \pm 0.57 \mathrm{nmol} / \mathrm{L})$. In contrast, the Addison group showed a non-significant difference between the mean values obtained by both methods (mean \pm SE $8.31 \pm 3.08$ vs $7.19 \pm 3.08$; $p=0.6$ ). Figure 1 shows a typical LC-MS/MS chromatogram of a healthy subject (Figure 1A), a mitotane treated patient (Figure 1B) and an $\mathrm{AD}$ patient (Figure 1C). As shown in the figure, no peaks are present at retention times which differ from the cortisol ones in patients with $\mathrm{AD}$, whereas several other peaks appear in the chromatogram of both mitotane treated patients and healthy subjects. Moreover, we performed a paired t-test between ECLIA and LC-MS/MS results in the different subgroups showing a $\mathrm{p}<0.01$ in the group of patients treated with mitotane and a statistically non-significant $\mathrm{p}$ value $(\mathrm{p}=0.63)$ in patients affected by Addison disease.

\section{DISCUSSION}

Mitotane is the main drug for ACC treatment, inducing steroidogenesis inhibition with adrenolytic effects. Although not conclusively proven, metabolic transformation and oxidative damage, induced by free radicals, are generally accepted as mechanisms that mediate mitotane cytotoxicity. ${ }^{3}$ Moreover, elevated doses of mitotane, administered as a steroidogenesis inhibitor, generate metabolites which bind macromolecules in adrenocortical cell mitochondria, finally leading to their destruction and cellular necrosis. ${ }^{17}$

Patients on mitotane should be monitored in order to adjust steroid replacement doses, but there are important considerations regarding the choice of the optimal test to perform. Mitotane leads to a rise both in CBG levels (resulting in artificially elevated serum cortisol values) and in glucocorticoids clearance, ${ }^{8}$ so patients eventually require a 2 to 3-fold increase in hydrocortisone doses to achieve adequate replacement. Plasma ACTH levels could be useful for assessing glucocorticoid replacement adequacy. However, these levels are pulsatile and are an indirect evaluation of circulating corticosteroids. Furthermore, ACC patients with long-standing hypercortisolism may present suppressed ACTH levels. UFC requires an accurate daily collection performed by the patient as difficulties in such collections are frequently reported, ${ }^{18}$ although it still represents an alternative. Moreover, UFC is affected by an increased metabolic clearance of glucocorticoids and is influenced by renal function. In such cases, neither serum cortisol nor ACTH nor UFC may be used to assess glucocorticoid sufficiency, suggesting the need for a different test. Finally, SC sampling is promising as samples are easy to obtain: this procedure does not require hospitalization and samples are stable up to 7 days at room temperature, and up to 1 month or more at $4{ }^{\circ} \mathrm{C} .{ }^{19} \mathrm{SC}$ reflects a free fraction of plasma cortisol and may thus be employed for a more accurate evaluation of patient monitoring during mitotane treatment. Indeed, salivary cortisol concentrations reflect unbound plasma cortisol concentrations and are therefore generally unaffected by variations in $\mathrm{CBG}$ values. 


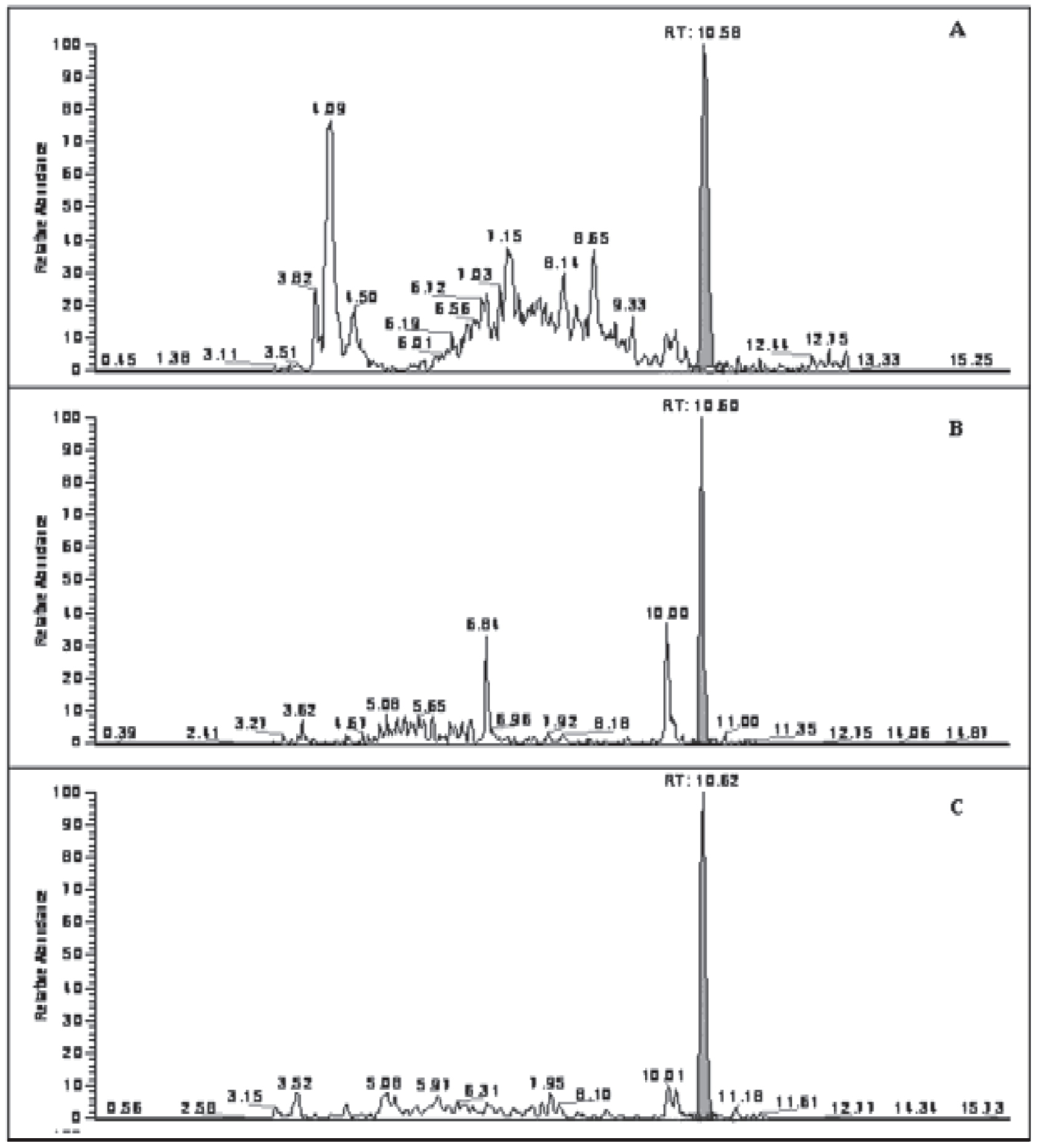

Figure 1. Typical HSRM chromatogram of salivary cortisol $(363.2 \rightarrow 121.1$ and $363.2 \rightarrow 327.0)$ of a control subject (A), of a subject treated with mitotane (B) and of an Addison disease subject (C).Cortisol retention time: $10.60 \mathrm{~min}$.

Several immunoassay methods have been proposed for the determination of SC, although cross-reactivity with endogenous cortisol precursors and metabolites significantly affected the results, especially for low concentration..$^{20}$ It should be noted that the measurement of SC by immunoassays gives higher concentrations compared to LC-MS/MS. These differences probably result from immunoassay cross-reactivities 
with endogenous steroids such as $6 \beta$-hydroxycortisol, 21-deoxycortisol, corticosterone but mainly with cortisol conjugated derivatives..$^{21,22}$ Indeed antibodies used with the ECLIA method significantly cross-react with similar steroid compounds. ${ }^{23}$

In our study, the comparison of SC values obtained by ECLIA and LC-MS/MS showed a significant overestimation of ECLIA results in patients on mitotane treatment and in healthy subjects. On the other hand, in $\mathrm{AD}$ patients, the values obtained between methods did not show statistically significant differences. In these subjects, in fact, only exogenous cortisol concentration (due to therapeutic intake) was determined by both methods, since steroidogenesis is completely absent and there are no steroidogenesis intermediates and cortisol-like analogues. In mitotane treated patients, drug steroidogenesis inhibition leads to the accumulation of intermediates and structurally similar steroids which may explain the falsely elevated cortisol results obtained by ECLIA. The same cross-reactivity, although less evident, is also present in healthy subjects, this probably related to the presence of the abovementioned molecules and confirming the overestimation of ECLIA with respect to LC-MS/MS. ${ }^{20}$ The analysis of LC-MS/ MS chromatograms represents another interesting issue (Figure 1). Steroid analysis by LC-MS/MS could be affected by the presence of possible interferences such as cortisol isomers or isobars, thus a very efficient chromatographic separation needs to be performed. In the chromatogram of healthy subjects (Figure 1A) and in that of patients treated with mitotane (Figure 1B), several peaks eluting at retention time different from the cortisol one confirm the presence of such interferences.

With our system it is not possible to identify these compounds but they probably have a steroid related structure (as fragmented in the same way). These compounds could in part explain the cross-reactivity of ECLIA vs LC-MS/MS. On the other hand, these peaks are not present in the chromatograms of patients with Addison (Figure 1C). The daily salivary cortisol profile for all patients parallels the normal circadian cortisol rhythm, which in turn reflects the hydrocortisone administration scheme.

In conclusion, salivary cortisol concentration seems to be a promising tool for mitotane patient monitor- ing. The preferential use of LC-MS/MS over ECLIA salivary cortisol measurements may be useful to monitor the steroid replacement therapy assays or for the screening for Cushing syndrome in the presence of any drug interference which may alter cortisol secretion and metabolism, besides mitotane, i.e. ketoconazole and anticonvulsants or other drugs inducing the liver P450 cytochrome enzyme system. ${ }^{24}$

SC, measured by LC-MS/MS, a highly specific, selective and interference-free method, allows specific hydrocortisone quantifications, excluding cortisol analogues that accumulate in patients in whom mitotane has not yet led to a complete adrenocytolitic effect. For this reason, LC-MS/MS offers a reliable system to monitor replacement therapy in mitotane treated patients.

\section{No grant was received.}

\section{REFERENCES}

1. Schteingart DE, Doherty GM, Gaucer PG, et al, 2005 Management of patients with adrenal cancer: recomendations of an international consensus conference. Endoc Relat Cancer 12: 667-680.

2. Terzolo M, Angeli A, Fassnacht M, et al, 2007 Adgiuvant mitotane treatment for adrenocortical carcinoma. N Engl J Med 356: 2372-2380.

3. Hahner S, Fassnacht M, 2005 Mitotane for adrenal carcinoma treatment. Curr Opin Investig Drugs 6: 386-394.

4. Young RB, Bryson MJ, Sweat ML, Street JC, 1973 Complexing of DDT and o,p'DDD with adrenal cytochrome P-450 hydroxylating systems. J Steroid Biochem 4: 585-591.

5. Trainer PJ, Besser M, 1994 Cushing's syndrome. Therapy directed at the adrenal glands. Endocrinol Metab Clin North Am 23: 571-584.

6. Løvås K, Curran S, Øksnes M, et al, 2010 Development of a disease-specific quality of life questionnaire in Addison's disease. J Clin Endocrinol Metab 95: 545-551.

7. Lacroix A, 2010 Approach to the patient with adrenocortical carcinoma. J Clin Endocrinol Metab 95: 4812-4822.

8. Bledsoe T, Island DP, Ney RL, Liddle GW, 1964 An effect of o,p-DDD on the extra-adrenal metabolism of cortisol in man. J Clin Endocrinol Metab 24: 1303-1311.

9. Van Seters AP, Moolenaar AJ, 1991 Mitotane increases the blood levels of hormone-binding proteins. Acta Endocrinolol (Copenh) 124: 526-533.

10. Gröschl M, 2008 Current status of salivary hormone analysis. Clin Chem 54: 1759-1769.

11. Daffara F, De Francia S, Reimondo G, et al, 2008 Prospective evaluation of mitotane toxicity in adrenocortical cancer patients treated adjuvantly. Endocr Relat Cancer 
15: 1043-1053.

12. Perogamvros I, Owen LJ, Newell-Price J, Ray DW, Trainer PJ, Keevil BG, 2009 Simultaneous measurement of cortisol and cortisone in human saliva using liquid chromatography-tandem mass spectrometry: application in basal and stimulated conditions. J Chromatogr B Analyt Technol Biomed Life Sci 877: 3771-3775.

13. Matsui F, Koh E, Yamamoto K, et al, 2009 Liquid chromatography-tandem mass spectrometry (LC-MS/ MS) assay for simultaneous measurement of salivary testosterone and cortisol in healthy men for utilization in the diagnosis of late-onset hypogonadism in males. Endocr J 56: 1083-1093.

14. Jönsson BA, Malmberg B, Amilon A, Helene Garde A, Orbaek P, 2003 Determination of cortisol in human saliva using liquid chromatography-electrospray tandem mass spectrometry. J Chromatogr B Analyt Technol Biomed Life Sci 784: 63-68.

15. Owen LJ, Haslam S, Adaway JE, Wood P, Glenn C, Keevil BG, 2010 A simplified liquid chromatography tandem mass spectrometry assay, using on-line solid-phase extraction, for the quantitation of cortisol in saliva and comparison with a routine DELFIA method. Ann Clin Biochem 47: 131-136.

16. Persichilli S, Gervasoni J, Iavarone F, Zuppi C, 2010 A simple liquid chromatography-tandem mass spectrometry method for urinary free cortisol analysis: suitable for routine purpose. Clin Chem Lab Med 48: 1433-1437.
17. Allolio B , Fassnacht M, 2006 Clinical review: adrenocortical carcinoma: clinical update. J Clin Endocrinol Metab 91: 2027-2037.

18. Nieman LK, Biller BM, Findling JW, et al, 2008 The diagnosis of Cushing's syndrome: an endocrine society clinical practice guideline. J Clin Endocrinol Metab 93: 1526-1540.

19. Carrozza C, Corsello SM, Paragliola RM, et al, 2010 Clinical accuracy of midnight salivarycortisol measured by automated electrochemiluminescence immunoassay method in Cushing's syndrome. Ann Clin Biochem 47: 228-232.

20. Raff H, Homar PJ , Burns EA, 2002 Comparison of two methods for measuring salivary cortisol. Clin Chem 48: 207-208.

21. Vogeser M, Durner J, Seliger E, Auernhammer C, 2006 Measurement of late-night salivary cortisol with an automated immunoassay system. Clin Chem Lab Med 44: 1441-1445.

22. Van Aken MO, Romijn JA, Miltenburg JA, Lentjes EG, 2003 Automated measurement of salivary cortisol. Clin Chem 49: 1408-1409.

23. Pearson Murphy BE, 1999 Lack of specificity of urinary free cortisol determinations: why does it continue? J Clin Endocrinol Metab 84: 2258-2259.

24. Putignano P, Kaltsas GA, Satta MA, Grossman AB, 1998 The effects of anti-convulsant drugs on adrenal function. Horm Metab Res. 30: 389-397. 